\title{
On Computing the Discrete Fourier Transform
}

\author{
By S. Winograd
}

\begin{abstract}
A new algorithm for computing the Discrete Fourier Transform is described. The algorithm is based on a recent result in complexity theory which enables us to derive efficient algorithms for convolution. These algorithms are then used to obtain the new Discrete Fourier Transform algorithm.
\end{abstract}

I. Introduction. A previous paper [1] investigated the minimum number of multiplications needed to obtain the coefficients of the product of two $(n-1)$ st degree polynomials modulo an $n$th degree polynomial. In this paper we will use the results of [1] to obtain new algorithms for computing the Discrete Fourier Transform (DFT). These new algorithms use about the same number of additions as the algorithms proposed by Cooley and Tukey [2], but only about $20 \%$ of the number of multiplications which their algorithm requires.

In the second section we will summarize the results needed for the construction of the algorithms. The third section will describe the derivation of the algorithms for cyclic convolutions, the fourth section will use these algorithms to derive the algorithm for DFT's of a few tens to a few thousands of points. In the last section we will discuss algorithms for multidimensional DFT's as well as algorithms for computing the DFT of very large numbers.

II. Theoretical Background. Let

$$
R_{l}(u)=\sum_{i=0}^{l} x_{i} u^{i}, \quad S_{m}(u)=\sum_{i=0}^{m} y_{i} u^{i}
$$

be two polynomials with indeterminate coefficients, and let $P(u)=u^{n}+\sum_{i=0}^{n-1} a_{i} u^{i}$ be a monic polynomial of degree $n$ with coefficients in a field $G$. (In the applications we will use $G$ as the field $Q$ of the rationals, only in the last section we will use other fields.) Assume $P(u)=P_{1}(u) \cdot P_{2}(u)$ such that $P_{1}(u)$ and $P_{2}(u)$ are relatively prime, and let $n_{1}=\operatorname{deg}\left(P_{1}\right)$ and $n_{2}=\operatorname{deg}\left(P_{2}\right)$.

Using the Chinese Remainder Theorem, we obtain:

$$
R_{l} \cdot S_{m} \bmod P
$$

$$
=\left(Q_{2} \cdot P_{2}\left(R_{l} \cdot S_{m} \bmod P_{1}\right)+Q_{1} \cdot P_{1} \cdot\left(R_{l} \cdot S_{m} \bmod P_{2}\right)\right) \bmod P,
$$

where $Q_{1}$ and $Q_{2}$ are polynomials such that

$$
Q_{1} P_{1}+Q_{2} \cdot P_{2}=1 \bmod P
$$

Received November 30, 1976 ; revised June 9, 1977.

AMS (MOS) subject classifications (1970). Primary 68A20. 
Let $\widetilde{T}$ be the set of coefficients of $R_{l} \cdot S_{m}$ and let $\widetilde{T}_{P}$ be the set of coefficients of $R_{l} \cdot S_{m}$ mod $P$. It was shown in [3] that at least $l+m+1$ multiplications are needed to compute $\widetilde{T}$ (multiplication by a fixed element $g \in G$ is not counted), and using the algorithm of [4] one can actually obtain an algorithm for computing $\widetilde{T}$ using $l+m+1$ multiplications. Clearly, we can obtain $\widetilde{T}_{P}$ from $\widetilde{T}$ using only additions and multiplications by elements $g \in G$; thus, the number of multiplications, which are counted, needed to compute $\widetilde{T}_{P}$ is at most $l+m+1$.

Another way of computing $\widetilde{T}_{P}$ when $P$ has more than one irreducible factor is by the use of the identity (1), i.e., if $P=P_{1} \cdot P_{2}$ such that $P_{1}$ and $P_{2}$ are relatively prime then we can use the algorithm for $\widetilde{T}_{P_{1}}$ to multiply $\left(R \bmod P_{1}\right) \cdot\left(S \bmod P_{1}\right)$ $\bmod P_{1}$, and the algorithm for $\widetilde{T}_{P_{2}}$ to multiply $\left(R \bmod P_{2}\right) \cdot\left(S \bmod P_{2}\right) \bmod P_{2}$, and then obtain the algorithm for $\widetilde{T}_{P}$ using only additional additions and multiplications by elements of $G$. It was shown in [1] that for the case $l=m=n-1$ the number of multiplications needed to compute $\widetilde{T}_{P}$ is $2 n-k$, where $k$ is the number of distinct irreducible factors of $P$. Moreover, every algorithm which computes $\widetilde{T}_{p}$ in $2 n-k$ multiplications uses (1).

When $P$ has only one irreducible factor, i.e., when $P$ is a power of irreducible polynomial, we cannot use (1); but then we compute $\widetilde{T}_{p}$ by computing $\widetilde{T}$ first and then reducing modulo $P$.

There are two ways for computing $\widetilde{T}$ using only $l+m+1$ multiplications. The first one uses the identity

$$
R_{l}(u) \cdot S_{m}(u)=R_{l}(u) \cdot S_{m}(u) \bmod \prod_{i=0}^{m+l}\left(u-\alpha_{i}\right)
$$

where the $\alpha_{i}$ 's are distinct elements of $G$. (We assume that $G$ is large enough. Actually, we will use in this paper only $G$ of characteristic 0 .) The right-hand side of (3) can be computed using (1) in $m+l+1$ multiplications. This algorithm is the same as the one described in [4].

A second algorithm uses the identity

$$
R_{l}(u) \cdot S_{m}(u)=R_{l}(u) \cdot S_{m}(u) \bmod \prod_{i=1}^{m+l}\left(u-\beta_{i}\right)+x_{l} y_{m} \prod_{i=1}^{m+l}\left(u-\beta_{i}\right)
$$

where the $\beta_{i}$ 's are distinct elements of $G$. It was shown in [1] that every algorithm for computing $\widetilde{T}$ in $m+l+1$ multiplication uses either (3) or (4).

At times it is desirable to avoid the constants which the algorithms of (3) or (4) necessitate, even at the expense of additional multiplication. One way of accomplishing this is to choose a polynomial $P(u)$ of degree $l+m+1$ with many distinct irreducible factors, but not necessarily only linear factors. Identity (3) is then modified to:

$$
R_{l}(u) \cdot S_{m}(u)=R_{l}(u) \cdot S_{m}(u) \bmod P .
$$

Similarly, we can modify identity (4).

Another theoretical development which will be needed in this paper is that of the dual or transpose of system. Let 


$$
\sum_{j=1}^{s} \sum_{i=1}^{r} a_{i j k} x_{i} y_{j}, \quad k=1,2, \ldots, t,
$$

be a system of bilinear forms, and assume that we have found an algorithm for computing this system using $n$ multiplications without using the commutative law. That is,

$$
\sum_{j=1}^{s} \sum_{i=1}^{r} a_{i j k} x_{i} y_{j}=\sum_{l=1}^{n} \gamma_{k, l}\left(\sum_{i=1}^{r} \alpha_{i, l} x_{i}\right)\left(\sum_{j=1}^{s} \beta_{j, l} y_{j}\right), \quad k=1,2, \ldots, t .
$$

Multiplying both sides of (7) by $z_{k}$ and summing over $k$, we obtain

$$
\sum_{k=1}^{t} \sum_{j=1}^{s} \sum_{i=1}^{r} a_{i j k} z_{k} x_{i} y_{j}=\sum_{l=1}^{n}\left(\sum_{k=1}^{t} \gamma_{k, z^{2}}\right)\left(\sum_{i=1}^{r} \alpha_{i, l} x_{i}\right)\left(\sum_{j=1}^{s} \beta_{j, l} y_{j}\right) .
$$

Equating the coefficients of $x_{i}$, we obtain

(9) $\sum_{k=1}^{t} \sum_{j=1}^{r} a_{i j k} z_{k} y_{j}=\sum_{l=1}^{n} \alpha_{i, l}\left(\sum_{k=1}^{t} \gamma_{k, l} z_{k}\right)\left(\sum_{j=1}^{s} \beta_{j, l} y_{j}\right), \quad i=1,2, \ldots, r$.

The left-hand side of (9) is called a dual (or transpose) system, and the right-hand side of (9) provides an algorithm for computing it using $n$ multiplications.

III. Cyclic Convolution. Consider the problem of computing the cyclic convolution of two sets of $n$ points $\left(x_{0}, x_{1}, \ldots, x_{n-1}\right)$ and $\left(y_{0}, y_{1}, \ldots, y_{n-1}\right)$. This can be written as

$$
\left(\begin{array}{cccc}
x_{0} & x_{1} \cdots & x_{n-2} & x_{n-1} \\
x_{1} & x_{2} \cdots & x_{n-1} & x_{0} \\
x_{3} & x_{4} \cdots & x_{0} & x_{1} \\
\cdot & & & \\
x_{n-1} & x_{0} \cdots & x_{n-3} & x_{n-2}
\end{array}\right)\left(\begin{array}{c}
y_{0} \\
y_{1} \\
\cdot \\
\cdot \\
y_{n-1}
\end{array}\right)
$$

It is readily verified that (10) is the system of coefficients of the polynomial

$$
\begin{aligned}
& \left(x_{0}+x_{1} u+x_{2} u^{2}+\cdots+x_{n-1} u^{n-1}\right) \\
& \left(y_{0}+y_{n-1} u+y_{n-2} u^{2}+\cdots+y_{1} u^{n-1}\right) \bmod u^{n}-1,
\end{aligned}
$$

and we can use the results described in the previous section to compute this system.

As an example we will take $n=3$, that is, we consider the system

$$
\left(\begin{array}{l}
z_{0} \\
z_{1} \\
z_{2}
\end{array}\right)=\left(\begin{array}{lll}
x_{0} & x_{1} & x_{2} \\
x_{1} & x_{2} & x_{0} \\
x_{2} & x_{0} & x_{1}
\end{array}\right)\left(\begin{array}{l}
y_{0} \\
y_{1} \\
y_{2}
\end{array}\right),
$$


which is the system of coefficients of

$$
\left(x_{0}+x_{1} u+x_{2} u^{2}\right)\left(y_{0}+y_{2} u+y_{1} u^{2}\right) \bmod \left(u^{3}-1\right)
$$

Since $u^{3}-1=(u-1)\left(u^{2}+u+1\right)$, we have to compute

$$
\begin{array}{r}
\left(x_{0}+x_{1} u+x_{2} u^{2}\right)\left(y_{0}+y_{2} u+y_{1} u^{2}\right) \bmod (u-1) \\
=\left(x_{0}+x_{1}+x_{2}\right)\left(y_{0}+y_{1}+y_{2}\right)
\end{array}
$$

and

$$
\left(x_{0}+x_{1} u+x_{2} u^{2}\right)\left(y_{0}+y_{2} u+y_{1} u^{2}\right) \bmod \left(u^{2}+u+1\right)
$$

$$
=\left(\left(x_{0}-x_{2}\right)+\left(x_{1}-x_{2}\right) u\right) \cdot\left(\left(y_{0}-y_{1}\right)+\left(y_{2}-y_{1}\right) u\right) \bmod \left(u^{2}+u+1\right) .
$$

The part of the computation (14) can be done in one multiplication. To compute (15) we first compute $\widetilde{T}$, that is, the coefficients of $\left(\left(x_{0}-x_{2}\right)+\left(x_{1}-x_{2}\right) u\right) \cdot$ $\left(\left(y_{0}-y_{1}\right)+\left(y_{2}-y_{1}\right) u\right)$. This is done using the identity:

$$
\begin{aligned}
& \left(\left(x_{0}-x_{2}\right)+\left(x_{1}-x_{2}\right) u\right)\left(\left(y_{0}-y_{1}\right)+\left(y_{2}-y_{1}\right) u\right) \\
& =\left(\left(x_{0}-x_{2}\right)+\left(x_{1}-x_{2}\right) u\right)\left(\left(y_{0}-y_{1}\right)+\left(y_{2}-y_{1}\right) u\right) \bmod u(u+1) \\
& \quad+\left(x_{1}-x_{2}\right)\left(y_{2}-y_{1}\right) u(u+1),
\end{aligned}
$$

which leads to the algorithm:

$$
\begin{aligned}
m_{1}= & \left(x_{0}-x_{2}\right)\left(y_{0}-y_{1}\right), \quad m_{2}=\left(x_{1}-x_{2}\right)\left(y_{2}-y_{1}\right), \\
m_{3}= & \left(\left(x_{0}-x_{2}\right)-\left(x_{1}-x_{2}\right)\right)\left(\left(y_{0}-y_{1}\right)-\left(y_{2}-y_{1}\right)\right)=\left(x_{0}-x_{1}\right)\left(y_{0}-y_{2}\right) . \\
& \left(x_{0}-x_{2}\right)\left(y_{0}-y_{1}\right)=m_{1}, \\
& \left(x_{0}-x_{2}\right)\left(y_{2}-y_{1}\right)+\left(x_{1}-x_{2}\right)\left(y_{0}-y_{1}\right)=m_{1}+m_{2}-m_{3}, \\
& \left(x_{1}-x_{2}\right)\left(y_{2}-y_{1}\right)=m_{2} .
\end{aligned}
$$

And consequently, the coefficients of (15) are

$$
m_{1}-m_{2} \text { and } m_{1}+m_{2}-m_{3}-m_{2}=m_{1}-m_{3} \text {. }
$$

Defining $m_{0}=\left(x_{0}+x_{1}+x_{2}\right)\left(y_{0}+y_{1}+y_{2}\right)$, we obtain

$$
\begin{gathered}
\left(x_{0}+x_{1} u+x_{2} u^{2}\right)\left(y_{0}+y_{2} u+y_{1} u^{2}\right) \bmod (u-1)=m_{0} \\
\left(x_{0}+x_{1} u+x_{2} u^{2}\right)\left(y_{0}+y_{2} u+y_{1} u^{2}\right) \bmod \left(u^{2}+u+1\right) \\
=\left(m_{1}-m_{2}\right)+\left(m_{1}-m_{3}\right) u
\end{gathered}
$$

and using (1), we obtain 


$$
\begin{aligned}
\left(x_{0}+\right. & \left.x_{1} u+x_{2} u^{2}\right)\left(y_{0}+y_{2} u+y_{1} u^{2}\right) \bmod \left(u^{3}-1\right) \\
= & \frac{u^{2}+u+1}{3} m_{0}+\left(\frac{-(u-1)(u+2)}{3}\right)\left(\left(m_{1}-m_{2}\right)+\left(m_{1}-m_{3}\right) u\right) \\
= & \left(\frac{m_{0}}{3}+\frac{m_{1}}{3}-\frac{2 m_{2}}{3}+\frac{m_{3}}{3}\right)+\left(\frac{m_{0}}{3}+\frac{m_{1}}{3}+\frac{m_{2}}{3}-\frac{2 m_{3}}{3}\right) u \\
& +\left(\frac{m_{0}}{3}-\frac{2 m_{1}}{3}+\frac{m_{2}}{3}+\frac{m_{3}}{3}\right) u^{2} .
\end{aligned}
$$

In many applications either the $x_{i}$ 's or the $y_{j}$ 's are known a priori, for example, they are the tap values; and therefore, computations involving only these variables can be done beforehand, and thus should not be counted. Assuming that the operations on the $x_{i}$ 's are not counted, we define

$$
m_{0}^{\prime}=\frac{x_{0}+x_{1}+x_{2}}{3}\left(y_{0}+y_{1}+y_{2}\right), \quad m_{1}^{\prime}=\frac{x_{0}-x_{2}}{3}\left(y_{0}-y_{1}\right),
$$

$$
m_{2}^{\prime}=\frac{x_{1}-x_{2}}{3}\left(y_{2}-y_{1}\right), \quad m_{3}^{\prime}=\frac{x_{0}-x_{1}}{3}\left(y_{0}-y_{2}\right)
$$

and the three desired quantities are:

(20b) $m_{0}^{\prime}+m_{1}^{\prime}-2 m_{2}^{\prime}+m_{3}^{\prime}, \quad m_{0}^{\prime}+m_{1}^{\prime}+m_{2}^{\prime}-2 m_{3}^{\prime}, \quad m_{0}^{\prime}-2 m_{1}^{\prime}+m_{2}^{\prime}+m_{3}^{\prime}$.

Another algorithm is obtained by noticing that the transpose of (12) is

$$
\left(\begin{array}{ccc}
z_{0} & z_{2} & z_{1} \\
z_{1} & z_{0} & z_{2} \\
z_{2} & z_{1} & z_{0}
\end{array}\right)\left(\begin{array}{l}
y_{0} \\
y_{1} \\
y_{2}
\end{array}\right)=\left(\begin{array}{ccc}
z_{0} & z_{1} & z_{2} \\
z_{1} & z_{2} & z_{0} \\
z_{2} & z_{0} & z_{1}
\end{array}\right)\left(\begin{array}{l}
y_{0} \\
y_{2} \\
y_{1}
\end{array}\right) .
$$

Transposing algorithm (20), we obtain

$$
\begin{array}{ll}
m_{0}=\frac{z_{0}+z_{1}+z_{2}}{3}\left(y_{0}+y_{1}+y_{2}\right), & m_{1}=\frac{z_{0}+z_{1}-2 z_{2}}{3}\left(y_{0}-y_{1}\right), \\
m_{2}=\frac{-2 z_{0}+z_{1}+z_{2}}{3}\left(y_{2}-y_{1}\right), & m_{3}=\frac{z_{0}-2 z_{1}+z_{2}}{3}\left(y_{0}-y_{2}\right) ;
\end{array}
$$

and the three quantities to be computed are:

$$
m_{0}+m_{1}+m_{3}, \quad m_{0}+m_{2}-m_{3}, \quad m_{0}-m_{1}-m_{2} .
$$

This method of obtaining simpler algorithms by transposing the system of bilinear forms is useful for other cyclic convolutions as well. Using the Chinese Remainder Theorem usually results in $Q_{1} \cdot P_{1}$ and $Q_{2} \cdot P_{2}$ coefficients other than $0,1,-1$, and transposing the algorithm results in moving these coefficients to what part which can be precomputed. 
The matrix in (10) can be viewed as the "multiplication table" for the group $z_{n}$ of addition modulo $n$. In case $n=n_{1} \cdot n_{2}$ where $n_{1}$ and $n_{2}$ are relatively prime, then $z_{n}$ is isomorphic to $z_{n_{1}} \times z_{n_{2}}$. Therefore, there exists a permutation of the rows and columns of the matrix of $(10)$ such that the resulting matrix can be partitioned into blocks of $n_{2} \times n_{2}$ cyclic matrices, and such that the blocks form an $n_{1} \times n_{1}$ cyclic matrix.

For example, since $6=2 \times 3$ we have the isomorphism

$$
\begin{array}{ll}
0 \rightarrow(0,0), & 1 \rightarrow(1,1), \quad 2 \rightarrow(0,2), \\
3 \rightarrow(1,0), & 4 \rightarrow(0,1), \quad 5 \rightarrow(1,2) ;
\end{array}
$$

and therefore, if we have the cyclic convolution

$$
\left(\begin{array}{l}
\psi_{0} \\
\psi_{1} \\
\psi_{2} \\
\psi_{3} \\
\psi_{4} \\
\psi_{5}
\end{array}\right)=\left(\begin{array}{llllll}
x_{0} & x_{1} & x_{2} & x_{3} & x_{4} & x_{5} \\
x_{1} & x_{2} & x_{3} & x_{4} & x_{5} & x_{0} \\
x_{2} & x_{3} & x_{4} & x_{5} & x_{0} & x_{1} \\
x_{3} & x_{4} & x_{5} & x_{0} & x_{1} & x_{2} \\
x_{4} & x_{5} & x_{0} & x_{1} & x_{2} & x_{3} \\
x_{5} & x_{0} & x_{1} & x_{2} & x_{3} & x_{4}
\end{array}\right)\left(\begin{array}{l}
y_{0} \\
y_{1} \\
y_{2} \\
y_{3} \\
y_{4} \\
y_{5}
\end{array}\right)
$$

and we arrange it in the order $0,4,2,3,1,5$ (that is, first those indices whose first coordinate is 0 and the second coordinate in ascending order, and then those indices whose first coordinate is 1 and the second coordinate in ascending order), we obtain

$$
\left(\begin{array}{c}
\psi_{0} \\
\psi_{4} \\
\psi_{2} \\
\hdashline \psi_{3} \\
\psi_{1} \\
\psi_{5}
\end{array}\right)=\left(\begin{array}{ccc:ccc}
x_{0} & x_{4} & x_{2} & x_{3} & x_{1} & x_{5} \\
x_{4} & x_{2} & x_{0} & x_{1} & x_{5} & x_{3} \\
x_{2} & x_{0} & x_{4} & x_{5} & x_{3} & x_{1} \\
\hdashline x_{3} & x_{1} & x_{5} & x_{0} & x_{4} & x_{2} \\
x_{1} & x_{5} & x_{3} & x_{4} & x_{2} & x_{0} \\
x_{5} & x_{3} & x_{1} & x_{2} & x_{0} & x_{4}
\end{array}\right)\left(\begin{array}{c}
y_{0} \\
y_{4} \\
y_{2} \\
\hdashline y_{3} \\
y_{1} \\
y_{5}
\end{array}\right),
$$

which is the same as (24), yet exhibits the block structure.

This block structure can be used to derive an algorithm by composing two different algorithms. Using $u^{2}-1=(u+1)(u-1)$, we immediately obtain:

$$
\left(\begin{array}{cc}
x_{0} & x_{1} \\
x_{1} & x_{0}
\end{array}\right)\left(\begin{array}{l}
y_{0} \\
y_{1}
\end{array}\right)=\left(\begin{array}{l}
\frac{x_{0}+x_{1}}{2}\left(y_{0}+y_{1}\right)+\frac{x_{0}-x_{1}}{2}\left(y_{0}-y_{1}\right) \\
\frac{x_{0}+x_{1}}{2}\left(y_{0}+y_{1}\right)-\frac{x_{0}-x_{1}}{2}\left(y_{0}-y_{1}\right)
\end{array}\right)
$$


for a cyclic convolution of two elements. If we define:

$$
\Phi_{0}=\left(\begin{array}{l}
\psi_{0} \\
\psi_{4} \\
\psi_{2}
\end{array}\right), \quad \Phi_{1}=\left(\begin{array}{l}
\psi_{3} \\
\psi_{1} \\
\psi_{5}
\end{array}\right), \quad Y_{0}=\left(\begin{array}{l}
y_{0} \\
y_{4} \\
y_{2}
\end{array}\right), \quad Y_{1}=\left(\begin{array}{l}
y_{3} \\
y_{1} \\
y_{5}
\end{array}\right),
$$

$$
X_{0}=\left(\begin{array}{lll}
x_{0} & x_{4} & x_{2} \\
x_{4} & x_{2} & x_{0} \\
x_{2} & x_{0} & x_{4}
\end{array}\right), \quad X_{1}=\left(\begin{array}{lll}
x_{3} & x_{1} & x_{5} \\
x_{1} & x_{5} & x_{3} \\
x_{5} & x_{3} & x_{1}
\end{array}\right)
$$

then we can write $(25)$ as

$$
\left(\begin{array}{l}
\Phi_{0} \\
\Phi_{1}
\end{array}\right)=\left(\begin{array}{ll}
X_{0} & X_{1} \\
X_{1} & X_{0}
\end{array}\right)\left(\begin{array}{l}
Y_{0} \\
Y_{1}
\end{array}\right)
$$

and using algorithm (26), we get

$$
\begin{array}{ll}
M_{1}=\frac{X_{0}+X_{1}}{2}\left(Y_{0}+Y_{1}\right), & M_{2}=\frac{X_{0}-X_{1}}{2}\left(Y_{0}-Y_{1}\right), \\
\Phi_{0}=M_{1}+M_{2}, & \Phi_{1}=M_{1}-M_{2} .
\end{array}
$$

Computing $M_{1}$ and $M_{2}$, we use algorithm (22). Thus we obtained an algorithm for (24) which uses eight multiplications and 34 additions. It should be noted that we could have factored 6 as $3 \times 2$ and obtained a different block structure, namely that of three point convolution of $2 \times 2$ blocks. In this case we would have obtained another algorithm for (24) using eight multiplications and 38 additions.

In Appendix A we give the algorithms derived for cyclic convolution of 2, 3, 4, 5 and 6 points. These algorithms are summarized in Table 1. The algorithm given for five point cyclic convolution does not use the minimum number of multiplications. Another algorithm could have been derived using only eight multiplications, but then the number of additions would have been much larger, and the constant coefficients would not have been $0, \pm 1$.

T ABLE I

\begin{tabular}{rrr}
\hline$n$ & \# mult. & \# add. \\
\hline 2 & 2 & 4 \\
3 & 4 & 11 \\
4 & 5 & 15 \\
5 & 10 & 31 \\
6 & 8 & 34 \\
\hline
\end{tabular}


IV. One Dimensional Fourier Transform. The Discrete Fourier Transform of $n$ points

$$
A_{k}=\sum_{j=0}^{n-1} w^{k j} a_{j}, \quad i=0,1, \ldots, n-1, \quad w=e^{2 \pi i / n},
$$

can be written as $\mathbf{A}=W$ a where $W_{i, j}=w^{i j}$. We will consider first the case that $n$ is a prime. In this case the matrix $\left.W\right|_{i, j \neq 0}$ can be viewed as the "multiplication table" for the group $M_{n}$ of nonzero integers relatively prime to $n$ with group operation of multiplication modulo $n$. As is well known, $M_{p^{r}} \cong z_{(p-1) p^{r-1}}$ for $p \neq 2$ a prime and $M_{2^{r}}$ $\cong z_{2} \times z_{2^{r-2}}$. That means that if $n$ is a prime, we can rearrange the rows and columns of $\left.W\right|_{i, j \neq 0}$ so the resulting matrix is cyclic. (The idea of rearranging the indices of the Discrete Fourier Transform of a prime number of points so as to obtain cyclic convolution was first suggested by C. M. Rader [5].) This is illustrated in (31) for the case $n=7$, i.e., (31) is another way of writing the Discrete Fourier Transform for seven points.

$$
\left(\begin{array}{l}
A_{0} \\
A_{1} \\
A_{3} \\
A_{2} \\
A_{6} \\
A_{4} \\
A_{5}
\end{array}\right)=\left(\begin{array}{ccccccc}
1 & 1 & 1 & 1 & 1 & 1 & 1 \\
1 & w^{1} & w^{3} & w^{2} & w^{6} & w^{4} & w^{5} \\
1 & w^{3} & w^{2} & w^{6} & w^{4} & w^{5} & w^{1} \\
1 & w^{2} & w^{6} & w^{4} & w^{5} & w^{1} & w^{3} \\
1 & w^{6} & w^{4} & w^{5} & w^{1} & w^{3} & w^{2} \\
1 & w^{4} & w^{5} & w^{1} & w^{3} & w^{2} & w^{6} \\
1 & w^{5} & w^{1} & w^{3} & w^{2} & w^{6} & w^{4}
\end{array}\right)\left(\begin{array}{l}
a_{0} \\
a_{1} \\
a_{3} \\
a_{2} \\
a_{6} \\
a_{4} \\
a_{5}
\end{array}\right), w=e^{2 \pi i / 7}
$$

We can now use the algorithm for six point cyclic convolution developed in the previous section to compute the seven point Fourier Transform. Actually, for later use, it is better to compute first $A_{i}-A_{0}, i=1,2, \ldots, 6$. (Note that we have not disturbed the symmetries and, therefore, can still use the algorithms developed in the previous section.) The resulting algorithm appears in Appendix B.

In case $n=p^{r}$ is a power of a prime number, the situation is very similar. We can permute the rows and columns of $W$ so as to have copies of $M_{p^{r}}, M_{p^{r-1}}, \ldots$, $M_{p 0}$. This permutation is best explained by means of an example. In (32) we illustrate the permutation of $W$ for a nine point Fourier Transform. The algorithm for the nine points Fourier Transform is in Appendix B.

$$
\left(\begin{array}{l}
A_{0} \\
A_{3} \\
A_{6} \\
A_{1} \\
A_{2} \\
A_{4} \\
A_{8} \\
A_{7} \\
A_{5}
\end{array}\right)=\left(\begin{array}{ccc:ccc:ccc}
1 & 1 & 1 & 1 & 1 & 1 & 1 & 1 & 1 \\
1 & 1 & 1 & w^{3} & w^{6} & w^{3} & w^{6} & w^{3} & w^{6} \\
1 & 1 & 1 & w^{6} & w^{3} & w^{6} & w^{3} & w^{6} & w^{3} \\
\hdashline 1 & w^{3} & w^{6} & w^{1} & w^{2} & w^{4} & w^{8} & w^{7} & w^{5} \\
1 & w^{6} & w^{3} & w^{2} & w^{4} & w^{8} & w^{7} & w^{5} & w^{1} \\
1 & w^{3} & w^{6} & w^{4} & w^{8} & w^{7} & w^{5} & w^{1} & w^{2} \\
\hdashline 1 & w^{6} & w^{3} & w^{8} & w^{7} & w^{5} & w^{1} & w^{2} & w^{4} \\
1 & w^{3} & w^{6} & w^{7} & w^{5} & w^{1} & w^{2} & w^{4} & w^{8} \\
1 & w^{6} & w^{3} & w^{5} & w^{1} & w^{2} & w^{4} & w^{8} & w^{7}
\end{array}\right)\left(\begin{array}{l}
a_{0} \\
a_{3} \\
a_{6} \\
a_{1} \\
a_{2} \\
a_{4} \\
a_{8} \\
a_{7} \\
a_{5}
\end{array}\right) .
$$


An examination of the algorithms for the seven and nine point Fourier Transform reveals that the multiplicand which depends on the powers of $w$ is either a real number or an imaginary number-never a general complex number. This is not a peculiarity of these two numbers, but a general property of these algorithms. In the case that $n=p^{r}$, $p \neq 2$, the group $M_{p^{r}}$ is isomorphic to $Z_{(p-1) p^{r-1}}$, and the element -1 of $M_{p^{r}}$ is mapped into $1 / 2(p-1) p^{r-1}$ under the isomorphism. But since $u^{(p-1) p^{r-1}}-1^{r}=$ $\left(u^{1 / 2(p-1) p^{r-1}}-1\right)\left(u^{1 / 2(p-1) p^{r-1}}+1\right)$, the part of the algorithm for cyclic convolution

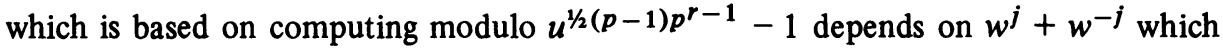
are real numbers; and the part of the algorithm which is based on $u^{1 / 2(p-1) p^{r-1}}+1$ depends on $w^{j}-w^{-j}$ which are imaginary numbers. A similar argument establishes this fact for $n=2^{r}$. Table 2 summarizes the algorithms for computing the Discrete Fourier Transform of $2,3,4,5,7,8,9$, and 16 points. The actual algorithms are given in Appendix B. Since we will later have to consider multiplication by $w^{0}=1$ as a multiplication, this is also summarized in Table 2.

TABLE 2

\begin{tabular}{rrrr}
\hline$n$ & \# Mult. & \# Mult. by $\boldsymbol{w}^{\mathbf{0}}$ & \# Add. \\
\hline 2 & 0 & 2 & 2 \\
3 & 2 & 1 & 6 \\
4 & 0 & 4 & 8 \\
5 & 5 & 1 & 17 \\
7 & 8 & 1 & 36 \\
8 & 2 & 6 & 26 \\
9 & 10 & 1 & 45 \\
16 & 10 & 8 & 74 \\
\hline
\end{tabular}

We now turn our attention to performing the Discrete Fourier Transform of $n$ points where $n$ is not a power of a prime. The idea of using the Chinese Remainder Theorem for "building up" an algorithm for computing the Discrete Fourier Transform of composite numbers originated with I. J. Good [6]. Since the way we "build up" the algorithm is somewhat different from Good's method, we will describe the whole process in detail.

Assume $n=n_{1} \cdot n_{2}$ where $n_{1}$ and $n_{2}$ are relatively prime. By the Chinese Remainder Theorem we can represent every integer $0 \leqslant i \leqslant n$ by the pair $\left(i_{1}, i_{2}\right)$ such that if $i$ is represented by $\left(i_{1}, i_{2}\right)$ and $j$ by $\left(j_{1}, j_{2}\right)$, then $i+j \bmod n$ is represented by $\left(i_{1}+j_{1} \bmod n, i_{2}+j_{2} \bmod n_{2}\right)$ and $i \cdot j \bmod n$ is represented by $\left(i_{1} \cdot j_{1} \bmod n\right.$, $\left.i_{2} \cdot j_{2} \bmod n_{2}\right)$.

Therefore, if we let $w$ be the $n$th root of unity then:

$$
\begin{aligned}
w^{k \cdot j} & =w^{k \cdot j \bmod n}=w^{\left(k_{1}, k_{2}\right)\left(j_{1}, j_{2}\right)}=w^{\left(k_{1} \cdot j_{1}, k_{2} \cdot j_{2}\right)} \\
& =w^{\left(k_{1} j_{1}, 0\right)+\left(0, k_{2} j_{2}\right)}=w^{\left(k_{1} j_{1}, 0\right)} w^{\left(0, k_{2} j_{2}\right)}=\left(w^{(1,0)}\right)^{k_{1} j_{1}} \cdot\left(w^{(0,1)}\right)^{k_{2} j_{2}} .
\end{aligned}
$$


That means that if we permute the rows and columns of the Discrete Fourier Transform matrix so as to arrange the indices to be in the lexicographical order of their representation, it can be partitioned in $n_{1} \times n_{1}$ blocks each of dimensions $n_{2} \times n_{2}$. The block in position $r, s$ will be $\left(w^{(1,0)}\right)^{r \cdot s} W_{2}$ when the $u, v$ entry of $W_{2}$ is $\left(w^{(0,1)}\right)^{u \cdot v}$. But $w^{(1,0)}$ is $w_{1}^{a}$ where $w_{1}$ is the $n_{1}$ th root of unity (note that the number represented by $(1,0)$ is divisible by $n_{2}$ ), and $w^{(0,1)}$ is $w_{2}^{b}$ where $w_{2}$ is the $n_{2}$ th root of unity. Consequently, $w_{2}$ is the same as the Discrete Fourier Transform matrix for $n_{2}$ points except that $w_{2}$ is replaced by $w_{2}^{b}$. If we denote by $w_{1}$ the matrix of the Discrete Fourier Transform of $n_{1}$ points where $w_{1}$ is replaced by $w_{1}^{a}$, then the matrix of Discrete Fourier Transform of $n_{1} \cdot n_{2}$ points has been transformed to the direct product of $W_{1}$ and $W_{2}$.

For example, take $n=12=3 \cdot 4$. The correspondence according to the Chinese Remainder Theorem is

$$
\begin{array}{llll}
0-(0,0) & 1-(1,1) & 2-(2,2) & 3-(0,3) \\
4-(1,0) & 5-(2,1) & 6-(0,2) & 7-(1,3) \\
8-(2,0) & 9-(0,1) & 10-(1,2) & 11-(2,3)
\end{array}
$$

and put in lexicographical order we get the rearrangement: $0,9,6,3,4,1,10,7,8,5,2$, 11. Thus the Discrete Fourier Transform for 12 points can be written as:

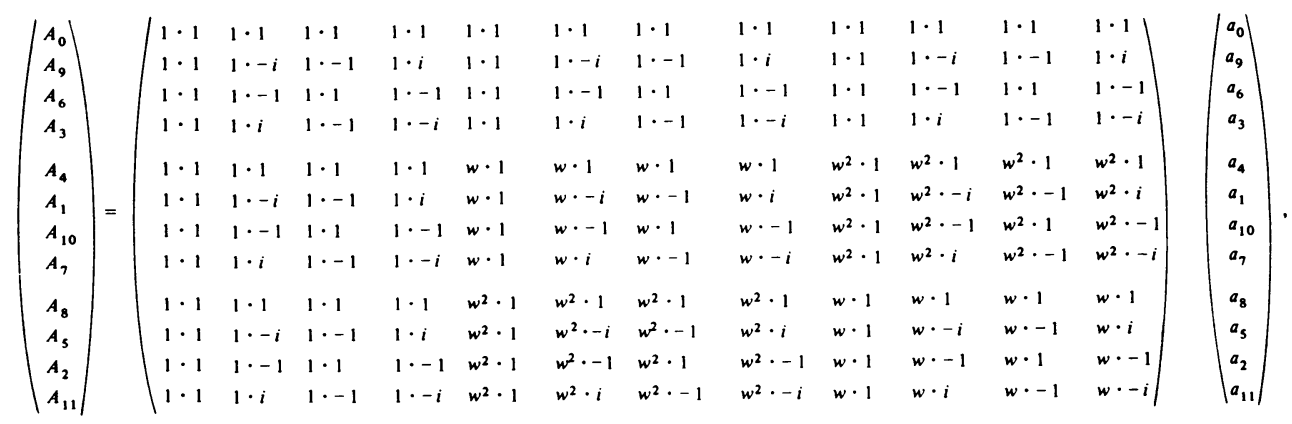

where $w$ is the cubic root of unity. Since $(1,0)$ corresponds to four, we have $a=1$, and since $(0,1)$ corresponds to nine we have $b=3$.

The decomposition of the 12 point Discrete Fourier Transform leads to an algorithm for its computation. If we define

$$
\begin{aligned}
& \mathrm{a}_{0}=\left(\begin{array}{c}
a_{0} \\
a_{9} \\
a_{6} \\
a_{3}
\end{array}\right), \quad \mathrm{a}_{1}=\left(\begin{array}{l}
a_{4} \\
a_{1} \\
a_{10} \\
a_{7}
\end{array}\right), \quad \mathrm{a}_{2}=\left(\begin{array}{l}
a_{8} \\
a_{5} \\
a_{2} \\
a_{11}
\end{array}\right), \\
& \mathbf{A}_{0}=\left(\begin{array}{c}
A_{0} \\
A_{9} \\
A_{6} \\
A_{3}
\end{array}\right), \quad \mathrm{A}_{1}=\left(\begin{array}{c}
A_{4} \\
A_{1} \\
A_{10} \\
A_{7}
\end{array}\right), \quad \mathbf{A}_{2}=\left(\begin{array}{c}
A_{8} \\
A_{5} \\
A_{2} \\
A_{11}
\end{array}\right),
\end{aligned}
$$

then using Algorithm B2 we obtain: 


$$
\begin{gathered}
M_{0}=W_{2} \cdot\left(\mathrm{a}_{0}+\mathrm{a}_{1}+\mathrm{a}_{2}\right), \quad M_{1}=\left(\cos \frac{2 \pi}{3}-1\right) W_{2} \cdot\left(\mathrm{a}_{1}+\mathrm{a}_{2}\right), \\
M_{2}=i \sin \frac{2 \pi}{3} W_{2} \cdot\left(\mathrm{a}_{1}-\mathrm{a}_{2}\right), \\
\mathbf{A}_{0}=M_{0}, \quad \mathbf{A}_{1}=M_{0}+M_{1}+M_{2}, \quad \mathbf{A}_{2}=M_{0}+M_{1}-M_{2},
\end{gathered}
$$

-where $W_{2}$ is the four point discrete Fourier Transform with $i$ replaced by $-i$ (since $b=3$ ). Therefore, we can use the Algorithm B3 to compute $M_{0}, M_{1}$, and $M_{2}$. In computing $M_{1}$, for example, we have to modify Algorithm B3 by first replacing $i$ by $-i$ and second multiplying the constants in the multiplication steps by $(\cos 2 \pi / 3-1)$. These modifications are done initially when we derive the algorithm and, therefore, are not counted in analyzing the computation complexity of the algorithm. In the end of this section we will show how one can avoid the first modification.

It should be clear that the way we derived the algorithm for computing the 12 points Discrete Fourier Transform is quite general. If $n=n_{1} \cdot n_{2}$ (where $n_{1}, n_{2}$ are relatively prime) and we have algorithms for computing the Discrete Fourier Transform of $n_{1}$ points using $a_{1}$ additions and $m_{1}$ multiplications, (including multiplication by 1) and of $n_{2}$ points using $a_{2}$ additions and $m_{2}$ multiplications, we can combine them to obtain an algorithm for computing the $n$ points Discrete Fourier Transform using $m_{1} \cdot m_{2}$ multiplications and $n_{2} \cdot a_{1}+m_{1} \cdot a_{2}$ additions. Since we would have decomposed the $n$ points discrete Fourier Transform using $n=n_{2} \cdot n_{1}$ as well, we could have derived an algorithm using $m_{1} \cdot m_{2}$ multiplications and $n_{1} \cdot a_{2}+m_{2} \cdot a_{1}$ additions. In general, these two algorithms will differ in their number of additions. In Table 3 we summarize the number of multiplications and additions used in algorithms derived this way for various values of $n$. For the sake of comparison with FFT we also tabulate $2 n \log _{2} n$ and

\begin{tabular}{|c|c|c|c|c|}
\hline$n$ & $\begin{array}{c}\text { \# Mult. } \\
\text { Complex Data }\end{array}$ & $\begin{array}{c}\text { \# Add. } \\
\text { Complex Data }\end{array}$ & $2 n \log _{2} n$ & $3 n \log _{2} n$ \\
\hline 30 & 72 & 384 & 295 & 442 \\
\hline 48 & 108 & 636 & 537 & 805 \\
\hline 60 & 144 & 888 & 709 & 1064 \\
\hline 120 & 288 & 2076 & 1658 & 2487 \\
\hline 168 & 432 & 3492 & 2484 & 3726 \\
\hline 240 & 648 & 5016 & 3796 & 5693 \\
\hline 420 & 1296 & 11352 & 7320 & 10980 \\
\hline 504 & 1584 & 14642 & 9050 & 13574 \\
\hline 840 & 2592 & 24804 & 16320 & 24480 \\
\hline 1008 & 3564 & 34920 & 20115 & 30172 \\
\hline 2520 & 9504 & 100188 & 56949 & 85423 \\
\hline
\end{tabular}
$3 n \log _{2} n$ (the formulas for the number of real multiplications and real additions, respectively, in FFT). 
As we saw before, one of the modifications needed to compute the Discrete Fourier Transform of $n_{1} \cdot n_{2}$ points is to replace $w_{1}$ by $w_{1}^{a}$ and $w_{2}$ by $w_{2}^{b}$. One way of avoiding this modification is to use different permutations on the rows and columns of the matrix, that is, different permutations of the input and output data.

Let $b_{0}, b_{1}, \ldots, b_{n-1}$ be the reordering of the input data $a_{0}, a_{1}, \ldots, a_{n-1}$; and let $B_{0}, B_{1}, \ldots, B_{n-1}$ be the reordering of the output data $A_{0}, A_{1}, \ldots, A_{n-1}$. Choose $r_{1}, r_{2}, s_{1}, s_{2}$ such that

$$
r_{1} \cdot s_{1} \cdot n_{1}=1 \bmod n_{2}, \quad r_{2} \cdot s_{2} \cdot n_{2}=1 \bmod n_{1} .
$$

If we choose $b_{j_{1} n_{2}+j_{2}}=a_{r_{1} j_{1} n_{1}+r_{2} j_{2} n_{2} \bmod n}$ and $B_{k_{1} n_{2}+k_{2}}=A_{s_{1} k_{1} n_{1}+s_{2} k_{2} n_{2} \bmod n}$, then the resulting entry of $\left(k_{1} n_{2}+k_{2}, j_{1} n_{2}+j_{2}\right)$ of the Discrete Fourier Transform matrix is (remember that $w^{n}=1$ ):

$$
\begin{aligned}
W_{\left(k_{1}, k_{2}\right)\left(j_{1}, j_{2}\right)} & =w^{\left(s_{1} k_{1} n_{1}+s_{2} k_{2} n_{2}\right)\left(r_{1} j_{1} n_{1}+r_{2} j_{2} n_{2}\right)} \\
& =w^{\left(s_{1} r_{1} n_{1}\right) k_{1} j_{1} n_{1}+\left(s_{2} r_{2} n_{2}\right) k_{2} j_{2} n_{2}} \\
& =\left(w^{n_{2}}\right)^{\left(s_{2} r_{2} n_{2}\right) k_{2} j_{2}} \cdot\left(w^{n_{1}}\right)^{\left(r_{1} s_{1} n_{1}\right) k_{1} j_{1}} \\
& =w_{1}^{\left(s_{2} r_{2} n_{2}\right) k_{2} j_{2}} \cdot w_{2}^{\left(r_{1} s_{1} n_{1}\right) k_{1} j_{1}} \\
& =w_{1}^{k_{2} j_{2}} \cdot w_{2}^{k_{1} j_{1}} \quad\left(w_{1}^{n_{1}}=1, w_{2}^{n}=1\right) .
\end{aligned}
$$

Therefore, this matrix is the direct product of the matrix for $n_{1}$ point Discrete Fourier Transform and $n_{2}$ point Discrete Fourier Transform.

One easy way of getting $r_{1}, r_{2}, s_{1}$ and $s_{2}$ is to choose $r_{1}=r_{2}=1$ and $s_{1}, s_{2}$ according to the Chinese Remainder Theorem.

We will end this section with the remark that the algorithms developed here can be used in conjunction with FFT. The identity behind FFT states that computing the Discrete Fourier Transform of $n_{1} \cdot n_{2}$ points $\left(n_{1}, n_{2}\right.$ are not necessarily relatively prime) can be done by first performing the Discrete Fourier Transform of $n_{1}$ points $n_{2}$ times, then one performs $\left(n_{1}-1\right) n_{2}$ complex multiplications, and then one performs $n_{1}$ times the Discrete Fourier Transform of $n_{2}$ points. It is, of course, possible to use the algorithms developed here in the first and third stages of the FFT identity.

V. Multidimensional Fourier Transform. For the sake of concreteness we will consider only two dimensional Fourier Transform, even though it should be clear that the results apply to all dimensions. The $n_{1} \times n_{2}$ points Discrete Fourier Transform is

$$
A_{k, k^{\prime}}=\sum_{j=0}^{n_{1}-1} \sum_{j^{\prime}=0}^{n_{2}-1} w_{1}^{k j} w_{2}^{k^{\prime} j^{\prime}} a_{j, j^{\prime}}, \quad 0 \leqslant k \leqslant n_{1}-1,0 \leqslant k^{\prime} \leqslant n_{2}-1 .
$$

It is apparent from (39) that the matrix for the Discrete Fourier Transform of $n_{1} \times n_{2}$ points is the direct product of the matrix of $n_{1}$ points by the matrix for $n_{2}$ points, and consequently the methods of the end of the last section are immediately 
applicable to multidimensional Discrete Fourier Transform. This can be stated even more strongly by noting that underlying the method for one dimensional Discrete Fourier Transform is the transformation to multidimensional transform.

The main results in this section consist of illustrating how the full strength of the investigation of a product of polynomials modulo a polynomial, and their dependence on the field of scalars, can be utilized. The discussion in the preceding paragraph indicates that the techniques to be described are applicable in the one dimensional case as well, but their exposition is simpler in the multidimensional case.

As was mentioned in Section II, the minimum number of multiplications needed to compute $\widetilde{T}_{p}$ is $2 n-k$ where $n$ is the degree of $P$ and $k$ is the number of distinct irreducible factors of $P$. By choosing a larger field of constants we can increase $k$ and thus decrease the number of multiplications. For example, $\widetilde{T}_{u^{4}-1}$ requires five multiplications over the field $Q$ of rationals, but only four over the field $Q(i)$. Similarly, $\widetilde{T}_{u^{6}-1}$ requires eight multiplications over $Q$, but only six over $Q\left(e^{2 \pi i / 3}\right)$. Recalling that $\widetilde{T}_{u^{4}-1}$ is the cyclic convolution of four points and $\widetilde{T}_{u^{6}-1}$ is the cyclic convolution of six points, we see that if somehow we could take advantage of the larger fields we could reduce the complexity of cyclic convolutions and, therefore, of the Discrete Fourier Transform.

One way of utilizing algorithms over fields which are algebraic extensions of the rationals is to use them in the situation that the Discrete Fourier Transform is to be performed on more than one set of data. For the sake of concreteness assume that we have two independent sets of data: $\left\{a_{0}^{(1)}, a_{1}^{(2)}, \ldots, a_{N-1}^{(2)}\right\}$ and $\left\{a_{0}^{(2)}, a_{1}^{(2)}, \ldots, a_{N-1}^{(2)}\right\}$. We can "group" them together as $\left\{a_{0}, a_{1}, \ldots, a_{n-1}\right\}$ where $a_{j}=\left(a_{j}^{(1)}, a_{j}^{(2)}\right)$. Assume that the field of constants we want to use is $Q(I)$, where $I^{2}=-1$, i.e., the field of Gaussian rationals.

We can transform the vectors $a_{j}$ into an algebra over $Q(I)$ by defining:

$$
\begin{aligned}
& \text { 1. }\left(a_{j}^{(1)}, a_{j}^{(2)}\right)+\left(a_{k}^{(1)}, a_{k}^{(2)}\right)=\left(a_{j}^{(1)}+a_{k}^{(1)}, a_{j}^{(2)}+a_{k}^{(2)}\right) \\
& I \cdot\left(a_{j}^{(1)}, a_{j}^{(2)}\right)=\left(-a_{j}^{(2)}, a_{j}^{(1)}\right) \\
& \left(a_{j}^{(1)}, a_{j}^{(2)}\right)\left(a_{k}^{(1)}, a_{k}^{(2)}\right)=\left(b^{(1)}, b^{(2)}\right), \\
& b^{(1)}=a_{j}^{(1)} a_{k}^{(1)}-a_{j}^{(2)} a_{k}^{(2)}=a_{j}^{(1)}\left(a_{k}^{(1)}+a_{k}^{(2)}\right)-\left(a_{j}^{(1)}+a_{j}^{(2)}\right) a_{k}^{(2)} \\
& b^{(2)}=a_{j}^{(1)} a_{k}^{(2)}+a_{j}^{(2)} a_{k}^{(1)}=a_{j}^{(1)}\left(a_{k}^{(1)}+a_{k}^{(2)}\right)-\left(a_{j}^{(1)}-a_{j}^{(2)}\right) a_{k}^{(1)}
\end{aligned}
$$

That is, we view the vector $a_{j}$ as standing for $a_{j}^{(1)}+I \cdot a_{j}^{(2)}$ where $I^{2}=-1$. This is of course possible whenever the number of independent sets of data is the same as the dimension of the extension field. We see that in this setting, multiplication by $I$ is not counted (it amounts to interchanging the components of the vector and changing one of the signs), while multiplication of two elements of the algebra amounts to three multiplications of the components plus a certain number of additions. 
The pairs of data could have been transformed into an algebra of $Q(\phi)$ where $\phi^{2}+\phi+1=0$, if instead of (40) we would have defined:

$$
\begin{aligned}
& \left(a_{j}^{(1)}, a_{j}^{(2)}\right)+\left(a_{k}^{(1)}, a_{k}^{(2)}\right)=\left(a_{j}^{(1)}+a_{k}^{(1)}, a_{j}^{(2)}+a_{k}^{(2)}\right) \\
& \phi \cdot\left(a_{j}^{(1)}, a_{j}^{(2)}\right)=\left(-a_{j}^{(2)}, a_{j}^{(1)}-a_{j}^{(2)}\right) \\
& \left(a_{j}^{(1)}, a_{j}^{(2)}\right) \cdot\left(a_{k}^{(1)}, a_{k}^{(2)}\right)=\left(b^{(1)}, b^{(2)}\right) \\
& b^{(1)}=\left(a_{j}^{(1)}+a_{j}^{(2)}\right) a_{k}^{(1)}-a_{j}^{(2)}\left(a_{k}^{(1)}-a_{k}^{(2)}\right) \\
& b^{(2)}=\left(a_{j}^{(1)} a_{k}^{(2)}+a_{j}^{(2)}\left(a_{k}^{(1)}-a_{k}^{(2)}\right) .\right.
\end{aligned}
$$

That is if we view the vector $a_{j}$ as standing for $a_{j}^{(1)}+\phi a_{j}^{(2)}$, where $\phi^{2}=-\phi-1$.

Having computed the Discrete Fourier Transform of the pair gives us the two desired Discrete Fourier Transforms.

As an example, consider performing the three dimensional Discrete Fourier Transform on $120 \times 120 \times 120$ data points, which are assumed to be complex. Using the method described in the beginning of this section would require 5,971,968 real multiplications and 97,203,456 real additions for each set of data. (For the sake of comparison with FFT, note that $2 \times 120^{3} \times \log _{2} 120^{3}=71,610,641$ and $3 \times 120^{3} \times$ $\log _{2} 120^{3}=107,415,962$.) Since $120=8 \times 3 \times 5$, we could have reduced the number of multiplications if we could have done the four point cyclic convolution (which appears in the five point Fourier Transform) in four multiplications instead of five. Since $Q(I)$ splits $u^{4}-1$, we choose to view the pair of input data as an algebra over $Q(I)$. In Appendix $\mathrm{C}$ we give an algorithm for the five point Fourier Transform over $Q(I)$. Using this algorithm, we can perform the $120 \times 120 \times 120$ Fourier Transform in $2 \times 5,184,000$ real multiplications and $2 \times 96,840,800$ real additions. But since this yields the results of performing the Fourier Transform on two sets of data, we obtained 13\% savings of the number of multiplications (and a slight reduction of the number of additions).

It should be clear that this construction is general. For example, computing the Discrete Fourier Transform of $252 \times 252$ points may be advantageously done over $Q(\phi)$; and the Fourier Transform of $140 \times 140 \times 140$ may be sped up by doing it over $Q(I, \phi)$.

Another, more subtle, way of utilizing the fact that $\widetilde{T}_{p}$ may require fewer multiplications when the field of constants is enlarged, is based on the construction in the beginning of [1].

The Chinese Remainder Theorem states that when $P_{1}$ and $P_{2}$ are relatively prime, the system $\widetilde{T}_{P_{1} \cdot P_{2}}$ can be transformed, by appropriate change of variables, to the direct sum of $\widetilde{T}_{P_{1}}$ and $\widetilde{T}_{P_{2}}$. We will illustrate this by considering the four point cyclic convolution, i.e., $\widetilde{T}_{u^{4}-1}$. Since $u^{4}-1=\left(u^{2}-1\right)\left(u^{2}+1\right)$, we obtain: 


$$
\left(\begin{array}{llll}
x_{1} & x_{2} & x_{3} & x_{4} \\
x_{2} & x_{3} & x_{4} & x_{1} \\
x_{3} & x_{4} & x_{1} & x_{2} \\
x_{4} & x_{1} & x_{2} & x_{3}
\end{array}\right)\left(\begin{array}{l}
y_{1} \\
y_{2} \\
y_{3} \\
y_{4}
\end{array}\right)=\left(\begin{array}{cccc}
1 & 0 & 0 & 1 \\
0 & 1 & 1 & 0 \\
1 & 0 & 0 & -1 \\
0 & 1 & -1 & 0
\end{array}\right)
$$

$$
\text { . }\left(\begin{array}{cccc}
\frac{x_{1}+x_{3}}{2} & \frac{x_{2}+x_{4}}{2} & 0 & 0 \\
\frac{x_{2}+x_{4}}{2} \frac{x_{1}+x_{3}}{2} & 0 & 0 \\
0 & 0 & \frac{x_{2}-x_{4}}{2} & -\frac{x_{1}-x_{3}}{2} \\
0 & 0 & \frac{x_{1}-x_{3}}{2} & \frac{x_{2}-x_{4}}{2}
\end{array}\right)\left(\begin{array}{l}
y_{1}+y_{3} \\
y_{2}+y_{4} \\
y_{1}-y_{3} \\
y_{2}-y_{4}
\end{array}\right) \text {. }
$$

This transformation can be carried directly into the appropriate Discrete Fourier Transform. Thus, the decomposition (42) translates into the following decomposition of the five point Discrete Fourier Transform

$$
\begin{aligned}
\left(\begin{array}{l}
A_{0} \\
A_{1} \\
A_{2} \\
A_{3} \\
A_{4}
\end{array}\right) & =\left(\begin{array}{ccccc}
1 & 0 & 0 & 0 & 0 \\
1 & 1 & 0 & 0 & 1 \\
1 & 0 & 1 & 1 & 0 \\
1 & 1 & 0 & 0 & -1 \\
1 & 0 & 1 & -1 & 0
\end{array}\right) \\
& \cdot\left(\begin{array}{ccccc}
1 & 0 & 0 & 0 & 0 \\
0 & \cos u-1 & \cos 2 u-1 & 0 & 0 \\
0 & \cos 2 u-1 & \cos u-1 & 0 & 0 \\
0 & 0 & 0 & i \sin 2 u & -i \sin u \\
0 & 0 & 0 & i \sin u & i \sin 2 u
\end{array}\right)\left(\begin{array}{c}
a_{0}+a_{1}+a_{2}+a_{3}+a_{4} \\
a_{1}+a_{4} \\
a_{2}+a_{3} \\
a_{1}-a_{4} \\
a_{2}-a_{3}
\end{array}\right) .
\end{aligned}
$$

If we consider the two dimensional Fourier Transform of $5 \times 5$ points it can be decomposed as

$$
\begin{gathered}
\left(\widetilde{T}_{u} \oplus \widetilde{T}_{u^{2}-1} \oplus \widetilde{T}_{u^{2}+1}\right) \otimes\left(\widetilde{T}_{u} \oplus \widetilde{T}_{u^{2}-1} \oplus \widetilde{T}_{u^{2}+1}\right) \\
=\widetilde{T}_{u} \oplus 2 \cdot \widetilde{T}_{u^{2}-1} \oplus 2 \cdot \widetilde{T}_{u^{2}+1} \oplus\left(\widetilde{T}_{u^{2}-1} \otimes \widetilde{T}_{u^{2}+1}\right) \\
\oplus 2 \cdot\left(\widetilde{T}_{u^{2}-1} \otimes \widetilde{T}_{u^{2}+1}\right) \oplus\left(\widetilde{T}_{u^{2}+1} \otimes \widetilde{T}_{u^{2}+1}\right)
\end{gathered}
$$

In [1] we showed how to compute $\widetilde{T}_{u^{2}+1} \otimes \widetilde{T}_{u^{2}+1}$ using six multiplications, and therefore the total number of multiplications needed to perform the $5 \times 5$ two dimensional Fourier Transform is $1+4+6+4+12+6=33$ (instead of 36). Using this construction to obtain an algorithm for the $5 \times 5 \times 5$ Fourier Transform, and 
then incorporating it in computing the $120 \times 120 \times 120$ points Fourier Transform we obtain an algorithm which uses $90,706,176$ real additions and $4,810,752$ real multiplications. (That is, $6.7 \%$ of the number of multiplications and $84 \%$ of the number of additions of FFT.)

It should be emphasized that the savings of the last construction occur at the expense of the length of the program. This construction calls for writing an algorithm to compute the $5 \times 5 \times 5$ Discrete Fourier Transform.

Acknowledgment. The author wishes to thank Dr. Ramesh C. Agarwal of IBM Research for helping in simplifying the algorithm for DFT of nine points.

\section{Appendix A}

A.

$$
\left(\begin{array}{l}
\psi_{1} \\
\psi_{2}
\end{array}\right)=\left(\begin{array}{ll}
x_{1} & x_{2} \\
x_{2} & x_{1}
\end{array}\right) \quad\left(\begin{array}{l}
y_{1} \\
y_{2}
\end{array}\right)
$$

Algorithm:

$$
\begin{array}{ll}
s_{1}=y_{1}+y_{2} & s_{2}=y_{1}-y_{2} \\
m_{1}=\frac{x_{1}+x_{2}}{2} \cdot s_{1} & m_{2}=\frac{x_{1}-x_{2}}{2} \cdot s_{2} \\
s_{3}=m_{1}+m_{2} & s_{4}=m_{1}-m_{2} \\
\psi_{1}=s_{3} & \psi_{4}=s_{4}
\end{array}
$$

A2.

$$
\left|\begin{array}{l}
\psi_{1} \\
\psi_{2} \\
\psi_{3}
\end{array}\right|=\left(\begin{array}{lll}
x_{1} & x_{2} & x_{3} \\
x_{2} & x_{3} & x_{1} \\
x_{3} & x_{1} & x_{2}
\end{array}|| \begin{array}{l}
y_{1} \\
y_{2} \\
y_{3}
\end{array} \mid\right.
$$

Algorithm:

$$
\begin{array}{lll}
s_{1}=y_{1}+y_{2} & s_{2}=y_{1}-y_{2} & s_{3}=y_{2}-y_{3} \\
s_{4}=y_{3}-y_{1} & s_{5}=y_{3}+s_{1} & \\
m_{1}=\frac{x_{1}+x_{2}+x_{3}}{3} \cdot s_{5} & m_{2}=\frac{2 x_{1}-x_{2}-x_{3}}{3} \cdot s_{2} & \\
m_{3}=\frac{x_{1}+x_{2}-2 x_{3}}{3} \cdot s_{3} & m_{4}=\frac{x_{1}-2 x_{2}+x_{3}}{3} \cdot s_{4} & s_{8}=m_{2}+m_{4} \\
s_{6}=m_{2}+m_{3} & s_{7}=m_{4}-m_{3} & s_{11}=m_{1}-s_{8} \\
s_{9}=m_{1}+s_{6} & s_{10}=m_{1}+s_{7} & \psi_{3}=s_{11} \\
\psi_{1}=s_{9} & \psi_{2}=s_{10} &
\end{array}
$$


A3.

$$
\left(\begin{array}{l}
\psi_{1} \\
\psi_{2} \\
\psi_{3} \\
\psi_{4}
\end{array}\right)=\left(\begin{array}{llll}
x_{1} & x_{2} & x_{3} & x_{4} \\
x_{2} & x_{3} & x_{4} & x_{1} \\
x_{3} & x_{4} & x_{2} & x_{2} \\
x_{4} & x_{1} & x_{2} & x_{3}
\end{array}\right)\left(\begin{array}{l}
y_{1} \\
y_{2} \\
y_{3} \\
y_{4}
\end{array}\right)
$$

Algorithm:

$$
\begin{aligned}
& s_{1}=y_{1}+y_{3} \quad s_{2}=y_{1}-y_{3} \quad s_{3}=y_{4}+y_{2} \quad s_{4}=y_{4}-y_{2} \\
& s_{5}=s_{1}+s_{3} \quad s_{6}=s_{1}-s_{3} \quad s_{7}=s_{2}+s_{4} \\
& m_{1}=\frac{x_{1}+x_{2}+x_{3}+x_{4}}{4} \cdot s_{5} \quad m_{2}=\frac{x_{1}-x_{2}+x_{3}-x_{4}}{4} \cdot s_{6} \\
& m_{3}=\frac{x_{1}+x_{2}-x_{3}-x_{4}}{2} \cdot s_{2} \quad m_{4}=\frac{x_{2}-x_{4}}{2} \cdot s_{7} \quad m_{5}=\frac{x_{1}-x_{2}-x_{3}+x_{4}}{2} \cdot s_{4} \\
& s_{8}=m_{1}+m_{2} \quad s_{9}=m_{1}-m_{2} \quad s_{10}=m_{3}-m_{4} \quad s_{11}=m_{4}+m_{5} \\
& s_{12}=s_{8}+s_{10} \quad s_{13}=s_{8}^{-s_{10}} \quad s_{14}=s_{9}+s_{11} \quad s_{15}=s_{9}^{-s_{11}} \\
& \psi_{1}=s_{12} \quad \psi_{2}=s_{14} \quad \psi_{3}=s_{13} \quad \psi_{4}=s_{15} \\
& \text { A4. } \\
& \left(\begin{array}{l}
\psi_{1} \\
\psi_{2} \\
\psi_{3} \\
\psi_{4} \\
\psi_{5}
\end{array}\right)=\left(\begin{array}{lllll}
x_{1} & x_{2} & x_{3} & x_{4} & x_{5} \\
x_{2} & x_{3} & x_{4} & x_{5} & x_{1} \\
x_{3} & x_{4} & x_{5} & x_{1} & x_{2} \\
x_{4} & x_{5} & x_{1} & x_{2} & x_{3} \\
x_{5} & x_{1} & x_{2} & x_{3} & x_{4}
\end{array}|| \begin{array}{l}
y_{1} \\
y_{2} \\
y_{3} \\
y_{4} \\
y_{5}
\end{array} \mid\right. \\
& s_{1}=y_{1}+y_{4} \quad s_{2}=y_{2}+y_{3} \quad s_{3}=s_{1}+s_{2} \quad s_{4}=s_{1}-s_{2} \quad s_{5}=s_{3}+y_{5} \\
& s_{6}=y_{1}-y_{5} \quad s_{7}=y_{1}-y_{2} \quad s_{8}=y_{2}-y_{5} \quad s_{9}=y_{1}-y_{3} \quad s_{10}=y_{2}-y_{4} \\
& s_{11}=y_{3}-y_{5} \quad s_{12}=y_{3}-y_{4} \quad s_{13}=y_{4}-y_{5} \\
& m_{0}=\frac{x_{1}+x_{2}+x_{3}+x_{4}+x_{5}}{5} \cdot s_{5} \quad m_{1}=\frac{x_{1}+x_{2}+x_{3}+x_{4}-4 x_{5}}{5} \cdot s_{6} \\
& m_{2}=\frac{2 x_{1}-3 x_{2}+2 x_{3}-3 x_{4}+2 x_{5}}{5} \cdot s_{7} \quad m_{3}=\frac{-4 x_{1}+x_{2}+x_{3}+x_{4}+x_{5}}{5} \cdot s_{8} \\
& m_{4}=\frac{2 x_{1}+2 x_{2}-3 x_{3}-3 x_{4}+2 x_{5}}{5} \cdot s_{9} \quad m_{5}=\frac{-x_{1}-x_{2}-x_{3}+2 x_{4}-x_{5}}{5} \cdot s_{4} \\
& m_{6}=\frac{2 x_{1}+2 x_{2}+2 x_{3}-3 x_{4}-3 x_{5}}{5} \cdot s_{10} \quad m_{7}=\frac{x_{1}-4 x_{2}+x_{3}+x_{4}+x_{5}}{5} \cdot s_{11} \\
& m_{8}=\frac{-3 x_{1}+2 x_{2}+2 x_{3}-3 x_{4}+2 x_{5}}{5} \cdot s_{12} \quad m_{9}=\frac{x_{1}+x_{2}-4 x_{3}+x_{4}+x_{5}}{5} \cdot s_{13}
\end{aligned}
$$




$$
\begin{aligned}
& s_{14}=m_{4}+m_{5} \quad s_{15}=m_{5}-m_{6} \quad s_{16}=m_{0}+m_{1} \quad s_{17}=s_{16}+m_{2} \\
& s_{18}=s_{17}+s_{14} \quad s_{19}=m_{0}-m_{2} \quad s_{20}=s_{19}+m_{3} \quad s_{21}=s_{20}-s_{15} \\
& s_{22}=m_{0}-s_{14} \quad s_{23}=s_{22}+m_{7} \quad s_{24}=s_{23}+m_{8} \quad s_{25}=m_{0}+s_{15} \\
& s_{26}=s_{25}-m_{8} \quad s_{27}=s_{26}+m_{9} \quad s_{28}=m_{0}-m_{1} \quad s_{29}=s_{28}-m_{3} \\
& s_{30}=s_{23}^{-m_{7}} \quad s_{31}=s_{30^{-m_{9}}} \\
& \nu_{1}=s_{18} \quad \psi_{2}=s_{21} \quad \psi_{3}=s_{24} \quad \psi_{4}=s_{27} \quad \psi_{5}=s_{31} \\
& \text { A5. }\left(\begin{array}{l}
v_{1} \\
v_{2} \\
v_{3} \\
v_{4} \\
v_{5} \\
v_{6}
\end{array}\right)=\left(\begin{array}{cccccc}
x_{1} & x_{2} & x_{3} & x_{4} & x_{5} & x_{6} \\
x_{2} & x_{3} & x_{4} & x_{5} & x_{6} & x_{1} \\
x_{3} & x_{4} & x_{5} & x_{6} & x_{1} & x_{2} \\
x_{4} & x_{5} & x_{6} & x_{1} & x_{2} & x_{3} \\
x_{5} & x_{6} & x_{1} & x_{2} & x_{3} & x_{4} \\
x_{6} & x_{1} & x_{2} & x_{3} & x_{4} & x_{5}
\end{array} \mid\left(\begin{array}{l}
y_{1} \\
y_{2} \\
y_{3} \\
y_{4} \\
y_{5} \\
y_{6}
\end{array} \mid\right.\right. \\
& s_{1}=y_{1}+y_{4} \quad s_{2}=y_{1}-y_{4} \quad s_{3}=y_{2}+y_{5} \quad s_{4}=y_{5}-y_{2} \\
& s_{5}=y_{3}+y_{6} \quad s_{6}=y_{3}-y_{6} \quad s_{7}=s_{1}+s_{3} \quad s_{8}=s_{7}+s_{5} \\
& s_{9}=s_{2}+s_{4} \quad s_{10}=s_{9}+s_{6} \quad s_{11}=s_{1}-s_{3} \quad s_{12}=s_{3}-s_{5} \\
& s_{13}=s_{5}-s_{1} \quad s_{14}=s_{2}-s_{4} \quad s_{15}=s_{4}-s \quad s_{16}=s_{6}-s_{2} \\
& m_{1}=\frac{x_{1}+x_{2}+x_{3}+x_{4}+x_{5}+x_{6}}{6} \cdot s_{8} \quad m_{2}=\frac{2 x_{1}-x_{2}-x_{3}+2 x_{4}-x_{5}-x_{6}}{6} \cdot s_{11} \\
& m_{3}=\frac{x_{1} x_{2}-2 x_{3}+x_{4}+x_{5}-2 x_{6}}{6} \cdot s_{12} \quad m_{4}=\frac{x_{1}-2 x_{2}+x_{3}+x_{4}-2 x_{5}+x_{6}}{6} \cdot s_{13} \\
& m_{5}=\frac{x_{1}-x_{2}+x_{3}-x_{4}+x_{5}-x_{6}}{6} \cdot s_{10} \quad m_{6}=\frac{2 x_{1}+x_{2}-x_{3}-2 x_{4}-x_{5}+x_{6}}{6} \cdot s_{14} \\
& m_{7}=\frac{x_{1}-x_{2}-2 x_{3}-x_{4}+x_{5}+2 x_{6}}{6} \cdot s_{15} \quad m_{8}=\frac{x_{1}+2 x_{2}+x_{3}-x_{4}-2 x_{5}-x_{6}}{6} \cdot s_{16} \\
& s_{17}=m_{1}+m_{2} \quad s_{18}=s_{17}+m_{3} \quad s_{19}=m_{1}-m_{3} \quad s_{20}=s_{19}+m_{4} \\
& s_{21}=m_{1}-m_{2} \quad s_{22}=s_{21}-m_{4} \quad s_{23}=m_{5}+m_{6} \quad s_{24}=s_{23}+m_{7} \\
& s_{25}=m_{5}-m_{7} \quad s_{26}=s_{25}+m_{8} \quad s_{27}=m_{5}-m_{6} \quad s_{28}=s_{27}-m_{8} \\
& s_{29}=s_{18}+s_{24} \quad s_{30}=s_{18}-s_{24} \quad s_{31}=s_{20}-s_{26} \quad s_{32}=s_{20}+s_{26} \\
& s_{33}=s_{22}+s_{28} \quad s_{34}=s_{22}-s_{28} \\
& \psi_{1}=s_{29} \quad \psi_{2}=s_{31} \quad \psi_{3}=s_{33} \quad \psi_{4}=s_{30} \quad \psi_{5}=s_{32} \quad \psi_{6}=s_{34}
\end{aligned}
$$




\section{Appendix B}

B1. $\left|\begin{array}{l}A_{0} \\ A_{1}\end{array}\right|=\left|\begin{array}{cc}w^{0} & w^{0} \\ w^{0} & -w^{0}\end{array}\right|\left|\begin{array}{l}a_{0} \\ a_{1}\end{array}\right| \quad w=e^{\frac{2 \pi i}{2}}=-1$

Algorithm:

$$
\begin{array}{ll}
s_{1}=a_{0}+a_{1} & s_{2}=a_{0}-a_{1} \\
m_{0}=1 \cdot s_{1} & m_{1}=1 \cdot s_{2} \\
A_{0}=m_{0} & A_{1}=m_{1}
\end{array}
$$

82. $\quad\left(\begin{array}{l}A_{0} \\ A_{1} \\ A_{2}\end{array}\right)=\left(\begin{array}{lll}w^{0} & w^{0} & w^{0} \\ w^{0} & w^{1} & w^{2} \\ w^{0} & w^{2} & w^{1}\end{array}\right)\left(\begin{array}{l}a_{0} \\ a_{1} \\ a_{2}\end{array}\right)$

Algorithm:

$$
\begin{array}{lll}
s_{1}=a_{1}+a_{2} & s_{2}=a_{1}-a_{2} & s_{3}=s_{1}+a_{0} \\
m_{0}=1 \cdot s_{3} & m_{1}=(\cos u-1) \cdot s_{1} & m_{2}=i \sin u \cdot s_{2} u=\frac{2 \pi}{3} \\
s_{4}=m_{0}+m_{1} & s_{5}=s_{4}+m_{2} & s_{6}=s_{4}-m_{2} \\
s_{0}=m_{0} & A_{1}=s_{5} & A_{2}=s_{6}
\end{array}
$$

B3. $\quad\left(\begin{array}{c}A_{0} \\ A_{1} \\ A_{2} \\ A_{3}\end{array}\right)=\left(\begin{array}{cccc}w^{0} & w^{0} & w^{0} & w^{0} \\ w^{0} & w^{1} & -w^{0} & -w^{1} \\ w^{0} & -w^{0} & w^{0} & -w^{0} \\ w^{0} & -w^{1} & -w^{0} & w^{1}\end{array}\right)\left(\begin{array}{l}a_{0} \\ a_{1} \\ a_{2} \\ a_{3}\end{array}\right)$

$s_{1}=a_{0}+a_{2}$

$$
s_{2}=a_{0}-a_{2}
$$

$$
s_{3}=a_{1}+a_{3}
$$$$
s_{4}=a_{1}-a_{3}
$$

$s_{5}=s_{1}+s_{3} \quad s_{6}=s_{1}-s_{3}$

$m_{1}=1 \cdot s_{5}$

$m_{2}=1 \cdot s_{6}$

$m_{3}=1 \cdot s_{2}$

$m_{4}=i \sin u \cdot s_{4}$

$u=\frac{2 \pi}{4}$

$s_{7}=m_{3}+m_{4} \quad s_{8}=m_{3}-m_{4}$

$A_{0}=m_{1}$

$A_{1}=s_{7}$

$A_{2}=m_{2}$

$A_{3}=s_{8}$ 
B4. $\quad\left(\begin{array}{l}A_{0} \\ A_{1} \\ A_{2} \\ A_{3} \\ A_{4}\end{array}\right)\left(\begin{array}{lllll}w^{0} & w^{0} & w^{0} & w^{0} & w^{0} \\ w^{0} & w^{1} & w^{2} & w^{3} & w^{4} \\ w^{0} & w^{2} & w^{4} & w^{1} & w^{3} \\ w^{0} & w^{3} & w^{1} & w^{4} & w^{2} \\ w^{0} & w^{4} & w^{3} & w^{2} & w^{1}\end{array}\right)\left(\begin{array}{l}a_{0} \\ a_{1} \\ a_{2} \\ a_{3} \\ a_{4}\end{array}\right) \quad w=e^{\frac{2 \pi i}{5}}$

Algorithm:

$$
\begin{aligned}
& s_{1}=a_{1}+a_{4} \quad s_{2}=a_{1}-a_{4} \quad s_{3}=a_{3}+a_{2} \quad s_{4}=a_{3}-a_{2} \\
& s_{5}=s_{1}+s_{3} \quad s_{6}=s_{1}-s_{3} \quad s_{7}=s_{2}+s_{4} \quad s_{8}=s_{5}+a_{0} \\
& m_{0}=1 \cdot s_{8} \quad m_{1}=\left(\frac{\cos u+\cos 2 u}{2}-1\right) \cdot s_{5} \quad m_{2}=\left(\frac{\cos u-\cos 2 u}{2}\right) \cdot s_{6} \quad u=\frac{2 \pi}{5} \\
& m_{3}=i(\sin u+\sin 2 u) \cdot s_{2} \quad m_{4}=i \sin 2 u \cdot s_{7} \quad m_{5}=i(\sin u-\sin 2 u) \cdot s_{4} \\
& s_{9}=m_{0}+m_{1} \quad s_{10}=s_{9}+m_{2} \quad s_{11}=s_{9}-m_{2} \quad s_{12}=m_{3}-m_{4} \\
& s_{13}=m_{4}+m_{5} \quad s_{14}=s_{10^{+}} s_{12} \quad s_{15}=s_{10^{-s}} \quad s_{16}=s_{11}+s_{13} \\
& s_{17}=s_{11} s_{13} \\
& A_{0}=m_{0} \quad A_{1}=s_{14} \quad A_{2}=s_{16} \quad A_{3}=s_{17} \quad A_{4}=s_{15} \\
& \text { B5. } \quad\left(\begin{array}{l}
A_{0} \\
A_{1} \\
A_{2} \\
A_{3} \\
A_{4} \\
A_{5} \\
A_{6}
\end{array}\right)=\left(\begin{array}{ccccccc}
w^{0} & w^{0} & w^{0} & w^{0} & w^{0} & w^{0} & w^{0} \\
w^{0} & w^{1} & w^{2} & w^{3} & w^{4} & w^{5} & w^{6} \\
w^{0} & w^{2} & w^{4} & w^{6} & w^{1} & w^{3} & w^{5} \\
w^{0} & w^{3} & w^{6} & w^{2} & w^{5} & w^{1} & w^{4} \\
w^{0} & w^{4} & w^{1} & w^{5} & w^{2} & w^{6} & w^{3} \\
w^{0} & w^{5} & w^{3} & w^{1} & w^{6} & w^{4} & w^{2} \\
w^{0} & w^{6} & w^{5} & w^{4} & w^{3} & w^{2} & w^{1}
\end{array}\right)\left(\begin{array}{l}
a_{0} \\
a_{1} \\
a_{2} \\
a_{3} \\
a_{4} \\
a_{5} \\
a_{6}
\end{array}\right)
\end{aligned}
$$

Algorithm:

$$
\begin{array}{llll}
s_{1}=a_{1}+a_{6} & s_{2}=a_{1}-a_{6} & s_{3}=a_{4}+a_{3} & s_{4}=a_{4}-a_{3} \\
s_{5}=a_{2}-a_{5} & s_{6}=a_{2}-a_{5} & s_{7}=s_{1}+s_{3} & s_{8}=s_{7}+s_{5} \\
s_{9}=s_{8}+a_{0} & s_{10}=s_{1}-s_{3} & s_{11}=s_{3}-s_{5} & s_{12}=s_{5}-s_{1} \\
s_{13}=s_{2}+s_{4} & s_{14}=s_{13}+s_{6} & s_{15}=s_{2}-s_{4} & s_{16}=s_{4}-s_{6} \\
s_{17}=s_{6}-s_{2} & m_{1}=\left(\frac{\cos u+\cos 2 u+\cos 3 u}{3}-1\right) \cdot s_{8} u=\frac{2 \pi i}{7} \\
m_{0}=1 \cdot s_{9} & m_{3}=\left(\frac{\cos u-2 \cos 2 u+\cos 3 u}{3}\right) \cdot s_{11} \\
m_{2}=\left(\frac{2 \cos u-\cos 2 u-\cos 3 u}{3}\right) \cdot s_{10} & m_{5}=i\left(\frac{\sin u+\sin 2 u-\sin 3 u}{3}\right) \cdot s_{14}
\end{array}
$$




$$
\begin{aligned}
& m_{6}=i\left(\frac{2 \sin u-\sin 2 u+\sin 3 u}{3}\right) \cdot s_{15} \quad m_{7}=i\left(\frac{\sin u-2 \sin 2 u-\sin 3 u}{3}\right) \cdot s_{16} \\
& m_{8}=i\left(\frac{\sin u+\sin 2 u+2 \sin 3 u}{3}\right) \cdot s_{17} \\
& s_{18}=m_{0}+m_{1} \quad s_{19}=s_{18}+m_{2} \quad s_{20}=s_{19}+m_{3} \quad s_{21}=s_{18}-m_{2} \\
& s_{22}=s_{21}-m_{4} \quad s_{23}=s_{18}{ }^{-m_{3}} \quad s_{24}=s_{23}+m_{4} \quad s_{25}=m_{5}+m_{6} \\
& s_{26}=s_{25}+m_{7} \quad s_{27}=m_{5}-m_{6} \quad s_{28}=s_{27}-m_{8} \quad s_{29}=m_{5}-m_{7} \\
& s_{30}=s_{29}+m_{8} \quad s_{31}=s_{20}+s_{26} \quad s_{32}=s_{20}-s_{26} \quad s_{33}=s_{22}+s_{28} \\
& s_{34}=s_{22}-s_{28} \quad s_{35}=s_{24}+s_{30} \quad s_{36}=s_{24}{ }^{-s_{30}} \\
& A_{0}=m_{0} \quad A_{1}=s_{31} \quad A_{2}=s_{33} \quad A_{3}=s_{36} \\
& A_{4}=s_{35} \quad A_{5}=s_{34} \quad A_{6}=s_{32}
\end{aligned}
$$

B6.

$$
\left(\begin{array}{l}
A_{0} \\
A_{1} \\
A_{2} \\
A_{3} \\
A_{4} \\
A_{5} \\
A_{6} \\
A_{7}
\end{array}\right)=\left(\begin{array}{llllllll}
w^{0} & w^{0} & w^{0} & w^{0} & w^{0} & w^{0} & w^{0} & w^{0} \\
w^{0} & w^{1} & w^{2} & w^{3} & w^{4} & w^{5} & w^{6} & w^{7} \\
w^{0} & w^{2} & w^{4} & w^{6} & w^{0} & w^{2} & w^{4} & w^{6} \\
w^{0} & w^{3} & w^{6} & w^{1} & w^{4} & w^{7} & w^{2} & w^{5} \\
w^{0} & w^{4} & w^{0} & w^{4} & w^{0} & w^{4} & w^{0} & w^{4} \\
w^{0} & w^{5} & w^{2} & w^{7} & w^{4} & w^{1} & w^{6} & w^{3} \\
w^{0} & w^{6} & w^{4} & w^{2} & w^{0} & w^{6} & w^{4} & w^{2} \\
w^{0} & w^{7} & w^{6} & w^{5} & w^{4} & w^{3} & w^{2} & w^{1}
\end{array}\right)\left(\begin{array}{l}
a_{0} \\
a_{1} \\
a_{2} \\
a_{3} \\
a_{4} \\
a_{5} \\
a_{6} \\
a_{7}
\end{array}\right) \quad w=e^{\frac{2 \pi i}{8}}
$$

Algorithm.

$$
\begin{array}{llll}
s_{1}=a_{0}+a_{4} & s_{2}=a_{0}^{-a_{4}} & s_{3}=a_{2}+a_{6} & s_{4}=a_{2}-a_{6} \\
s_{5}=a_{1}+a_{5} & s_{6}=a_{1}-a_{5} & s_{7}=a_{3}+a_{7} & s_{8}=a_{3}-a_{7} \\
s_{9}=s_{1}+s_{3} & s_{10}=s_{1}-s_{3} & s_{11}=s_{5}+s_{7} & s_{12}=s_{5}-s_{7} \\
s_{13}=s_{9}+s_{11} & s_{14}=s_{9}-s_{11} & s_{15}=s_{6}+s_{8} & s_{16}=s_{6}-s_{8} \\
m_{1}=1 \cdot s_{13} & m_{2}=1 \cdot s_{14} \quad m_{3}=1 \cdot s_{10} \quad m_{4}=i \sin 2 u \cdot s_{12} u=\frac{2 \pi}{8} \\
m_{5}=1 \cdot s_{2} \quad m_{6}=i \cdot s i n 2 u_{4} s_{4} & m_{7}=i \sin u \cdot s_{15} & m_{8}=\cos u \cdot s_{16} \\
s_{17}=m_{3}+m_{4} & s_{18}=m_{3}-m_{4} & s_{19}=m_{5}+m_{8} & s_{20}=m_{5}-m_{8} . \\
s_{21}=m_{6}+m_{7} & s_{22}=m_{6}-m_{7} & s_{23}=s_{19}+s_{21} & s_{24}=s_{19^{-s}} \\
s_{25}=s_{20}+s_{22} & s_{26}=s_{20}-s_{22} &
\end{array}
$$




$$
\begin{aligned}
& A_{0}=m_{1} \\
& \left.A_{4}=m_{2}=s_{23} \quad \begin{array}{c}
A_{2}=s_{17} \\
A_{5}=s_{25} \\
B^{\prime} \\
A_{1} \\
A_{2} \\
A_{3} \\
A_{4} \\
A_{5} \\
A_{6} \\
A_{7} \\
A_{8}
\end{array}\right)=\left(\begin{array}{lllllllll}
A_{0} & w^{0} & w^{0} & w^{0} & w^{0} & w^{0} & w^{0} & w^{0} & w^{0} \\
w^{0} & w^{1} & w^{2} & w^{3} & w^{4} & w^{5} & w^{6} & w^{7} & w^{8} \\
w^{0} & w^{2} & w^{4} & w^{6} & w^{8} & w^{1} & w^{3} & w^{5} & w^{7} \\
w^{0} & w^{3} & w^{6} & w^{0} & w^{3} & w^{6} & w^{0} & w^{3} & w^{6} \\
w^{0} & w^{4} & w^{8} & w^{3} & w^{7} & w^{2} & w^{6} & w^{1} & w^{5} \\
w^{0} & w^{5} & w^{1} & w^{6} & w^{2} & w^{7} & w^{3} & w^{8} & w^{4} \\
w^{0} & w^{6} & w^{3} & w^{0} & w^{6} & w^{3} & w^{0} & w^{6} & w^{3} \\
w^{0} & w^{7} & w^{5} & w^{3} & w^{1} & w^{8} & w^{6} & w^{4} & w^{2} \\
w^{0} & w^{8} & w^{7} & w^{6} & w^{5} & w^{4} & w^{3} & w^{2} & w^{1} \\
a^{1} \\
a_{2} \\
a_{2} \\
a_{3} \\
a_{4} \\
a_{5} \\
a_{6} \\
a_{7} \\
a_{8}
\end{array}\right)
\end{aligned}
$$

\section{Algorithm:}

$$
\begin{aligned}
& s_{1}=a_{1}+a_{8} \quad s_{2}=a_{1}-a_{8} \quad s_{3}=a_{7}+a_{2} \quad s_{4}=a_{7}-a_{2} \\
& s_{5}=a_{3}+a_{6} \quad s_{6}=a_{3}-a_{6} \quad s_{7}=a_{4}+a_{5} \quad s_{8}=a_{4}-a_{5} \\
& s_{9}=s_{1}+s_{3} \quad s_{10}=s_{9}+s_{7} \quad s_{11}=s_{10}+s_{5} \quad s_{12}=s_{11}+a_{0} \\
& s_{13}=s_{2}+s_{4} \quad s_{14}=s_{13}+s_{8} \quad s_{15}=s_{1}-s_{3} \quad s_{16}=s_{3}-s_{7} \\
& s_{17}=s_{7}-s_{1} \quad s_{18}=s_{2}-s_{4} \quad s_{19}=s_{4}-s_{8} \quad s_{20}=s_{8}-s_{2} \\
& m_{0}=1 \cdot s_{12} \quad m_{1}=\left(-\frac{1}{2}\right) \cdot s_{10} \quad m_{2}=i \sin 3 u \cdot s_{14} \quad u=\frac{2 \pi}{9} \\
& m_{3}=(\cos 3 u-1) \cdot s_{5} \quad m_{4}=i \sin 3 u \cdot s_{6} \quad m_{5}=\left(\frac{2 \cos u-\cos 2 u-\cos 4 u}{3}\right) \cdot s_{15} \\
& m_{6}=\left(\frac{\cos u+\cos 2 u-2 \cos 4 u}{3}\right) \cdot s_{16} \quad m_{7}=\left(\frac{\cos u-2 \cos 2 u+\cos 4 u}{3}\right) \cdot s_{17} \\
& m_{8}=i\left(\frac{2 \sin u+\sin 2 u-\sin 4 u}{3}\right) \cdot s_{18} \quad 1 \quad m_{9}=i\left(\frac{\sin u-\sin 2 u-2 \sin 4 u}{3}\right) \cdot s_{19} \\
& m_{10}=i\left(\frac{\sin u+2 \sin 2 u+\sin 4 u}{3}\right) \cdot s_{20} \\
& \begin{array}{llll}
s_{21}=m_{1}+m_{1} & s_{22}=s_{20}+m_{1} & s_{23}=m_{0}+s_{22} & s_{24}=s_{23} m_{2} \\
s_{25}=s_{23}-m_{2} & s_{26}=m_{0}+m_{3} & s_{27}=s_{26}+s_{21} & s_{28}=s_{27}+m_{5} \\
s_{29}=s_{28}+m_{6} & s_{30}=s_{27}-m_{6} & s_{31}=s_{30}+m_{7} & s_{32}=s_{27}-m_{5} \\
s_{33}=s_{32}-m_{7} & s_{34}=m_{4}+m_{8} & s_{35}=s_{34}+m_{9} & s_{36}=m_{4}-m_{9} \\
s_{37}=s_{36}+m_{10} & s_{38}=m_{4}-m_{8} & s_{39}=s_{38}-m_{10} & s_{40}=s_{29}+s_{35} \\
s_{41}=s_{29}-s_{35} & s_{42}=s_{31}+s_{37} & s_{43}=s_{31}-s_{37} & s_{44}=s_{33}+s_{39} \\
s_{45}=s_{33}-s_{39} & & &
\end{array}
\end{aligned}
$$




$$
\begin{array}{ll}
A_{0}=m_{0} & A_{1}=s_{40} \quad A_{2}=s_{43} \quad A_{3}=s_{24} \quad A_{4}=s_{43} \\
A_{5}=s_{45} & A_{6}=s_{23} \quad A_{7}=s_{42} \quad A_{8}=s_{41} \\
\text { B8. } & A_{k}=\sum_{j=0}^{15} w^{k j} a_{j} \quad k=0,1, \ldots, 15 \quad w=e^{\frac{2 \pi i}{16}} .
\end{array}
$$

Algorithm.

$$
\begin{array}{llll}
s_{1}=a_{0}+a_{8} & s_{2}=a_{0}-a_{8} & s_{3}=a_{4}+a_{12} & s_{4}=a_{4}-a_{12} \\
s_{5}=a_{2}+a_{10} & s_{6}=a_{2}-a_{10} & s_{7}=a_{6}+a_{14} & s_{8}=a_{6}-a_{14} \\
s_{9}=a_{1}+a_{9} & s_{10}=a_{1}-a_{9} & s_{11}=a_{5}+a_{13} & s_{12}=a_{5}-a_{13} \\
s_{13}=a_{3}+a_{11} & s_{14}=a_{3}-a_{11} & s_{15}=a_{7}+a_{15} & s_{16}=a_{7}-a_{15} \\
s_{17}=s_{1}+s_{3} & s_{18}=s_{1}-s_{3} & s_{19}=s_{5}+s_{7} & s_{20}=s_{5}-s_{7} \\
s_{21}=s_{9}+s_{11} & s_{22}=s_{9}^{-s_{11}} & s_{23}=s_{13}+s_{15} & s_{24}=s_{13^{-s}} \\
s_{25}=s_{17^{+s}} & s_{26}=s_{17^{-s}} & s_{27}=s_{21}+s_{23} & s_{28}=s_{21}-s_{23} \\
s_{29}=s_{25} s_{27} & s_{30}=s_{25^{-s}} & s_{31}=s_{22}+s_{24} & s_{32}=s_{22}-s_{24} \\
s_{33}=s_{6}+s_{8} & s_{34}=s_{6}^{-s_{8}} & s_{35}=s_{10}+s_{16} & s_{36}=s_{10^{-s}} \\
s_{37}=s_{12}+s_{14} & s_{38}=s_{12}-s_{14} & s_{39}=s_{35}+s_{37} & s_{40}=s_{36}+s_{38}
\end{array}
$$

$$
\begin{aligned}
& m_{1}=1 \cdot s_{29} \quad m_{2}=1 \cdot s_{30} \quad m_{3}=1 \cdot s_{26} \quad m_{4}=i \sin 4 u \cdot s_{28} \quad u=\frac{2 \pi}{16} \\
& m_{5}=1 \cdot s_{18} \quad m_{6}=i \sin 4 u \cdot s_{20} \quad m_{7}=i \sin 2 u \cdot s_{31} \quad m_{8}=\cos 2 u \cdot s_{32} \\
& m_{9}=1 \cdot{ }_{2} \quad m_{10}=i \sin 4 u \cdot{ }_{4} \quad m_{11}=i \sin 2 u \cdot{ }_{33} \quad m_{12}=\cos 2 u \cdot s_{34} \\
& m_{13}=i \sin 3 u \cdot s_{39} \quad m_{14}=i(\sin u-\sin 3 u) \cdot s_{35} \quad m_{15}=i(\sin u+\sin 3 u) \cdot s_{37} \\
& m_{16}=\cos 3 u \cdot s_{40} \quad m_{17}=(\cos u+\cos 3 u) \cdot s_{36} \quad m_{18}=(\cos 3 u-\cos u) \cdot s_{38} \\
& s_{41}=m_{3}+m_{4} \quad s_{42}=m_{3}-m_{4} \quad s_{43}=m_{5}+m_{7} \quad s_{44}=m_{5}-m_{7} \\
& s_{45}=m_{6}+m_{8} \quad s_{46}=m_{6}-m_{8} \quad s_{47}=s_{43}+s_{45} \quad s_{48}=s_{43}-s_{45} \\
& s_{49}=s_{44}+s_{46} \quad s_{50}=s_{44}-s_{46} \quad s_{51}=m_{9}+m_{12} \quad s_{52}=m_{9}-m_{12} \\
& s_{53}=m_{10}+m_{11} \quad s_{54}=m_{10}-m_{11} \quad s_{55}=m_{13}+m_{14} \quad s_{56}=m_{13}-m_{15} \\
& s_{57}=m_{17}-m_{16} \quad s_{58}=m_{18}-m_{16} \quad s_{59}=s_{51}+s_{55} \quad s_{60}=s_{51}-s_{55} \\
& s_{61}=s_{52}+s_{56} \quad s_{62}=s_{52}-s_{56} \quad s_{63}=s_{53}+s_{57} \quad s_{64}=s_{53}-s_{57} \\
& s_{65}=s_{54}+s_{58} \quad s_{66}=s_{54}-s_{58} \quad s_{67}=s_{59}+s_{63} \quad s_{68}=s_{59}-s_{63}
\end{aligned}
$$


198

S. WINOGRAD

$$
\begin{aligned}
& s_{69}=s_{60}+s_{64} \quad s_{70}=s_{60}-s_{64} \quad s_{71}=s_{61}+s_{65} \quad s_{72}=s_{61}-s_{65} \\
& s_{73}=s_{62}+s_{66} \quad s_{74}=s_{62}-s_{66} \\
& A_{0}=m_{1} \quad A_{1}=s_{67} \quad A_{2}=s_{47} \quad A_{3}=s_{72} \quad A_{4}=s_{41} \quad A_{5}=s_{71} \\
& A_{6}=s_{48} \quad A_{7}=s_{68} \quad A_{8}=m_{2} \quad A_{9}=s_{69} \quad A_{10}=s_{49} \quad A_{11}=s_{74} \\
& A_{12}=s_{42} \quad A_{13}=s_{73} \quad A_{14}=s_{50} \quad A_{15}=s_{70}
\end{aligned}
$$

Appendix C

c1. $\left(\begin{array}{l}v_{1} \\ v_{2} \\ v_{3} \\ v_{4}\end{array}\right)=\left(\begin{array}{llll}x_{1} & x_{2} & x_{3} & x_{4} \\ x_{2} & x_{3} & x_{4} & x_{1} \\ x_{3} & x_{4} & x_{1} & x_{2} \\ x_{4} & x_{1} & x_{2} & x_{3}\end{array}\right)\left|\begin{array}{l}y_{1} \\ y_{2} \\ y_{3} \\ y_{4}\end{array}\right|$.

Algorithm:

$$
\begin{aligned}
& s_{1}=y_{1}+y_{3} \quad s_{2}=y_{1}-y_{3} \quad s_{3}=y_{4}+y_{2} \quad s_{4}=y_{4}-y_{2} \\
& s_{5}=s_{1}+s_{3} \quad s_{6}=s_{3}-s_{3} \quad s_{7}=s_{2}+I \cdot s_{4} \quad s_{8}=s_{2}-I \cdot s_{4} \\
& m_{1}=\frac{x_{1}+x_{2}+x_{3}+x_{4}}{4} \cdot s_{5} \quad m_{2}=\frac{x_{1}-x_{2}+x_{3}-x_{4}}{4} \cdot s_{6} \\
& m_{3}=\frac{\left(x_{1}-x_{3}\right)+I\left(x_{2}-x_{4}\right)}{4} \cdot s_{7} \quad m_{4}=\frac{\left(x_{1}-x_{3}\right)-I\left(x_{2}-x_{4}\right)}{4} \cdot s_{8} \\
& s_{9}=m_{1}+m_{2} \\
& s_{10}=m_{1}-m_{2} \\
& s_{11}=m_{4}+m_{3} \\
& s_{12}=m_{4}-m_{3} \\
& s_{13}=s_{9}+s_{11} \\
& s_{14}=s_{9}-s_{11} \\
& s_{15}=s_{10}+I \cdot s_{12} \\
& s_{16}=s_{10}-I \cdot s_{12} \\
& v_{1}=s_{13} \\
& \psi_{2}=s_{15} \\
& \psi_{3}=s_{14} \\
& \psi_{4}={ }^{\prime}{ }_{16} \\
& \left(\begin{array}{l}
A_{0} \\
A_{1} \\
A_{2} \\
A_{3} \\
A_{4}
\end{array}\right)=\left(\begin{array}{ccccc}
w^{0} & w^{0} & w^{0} & w^{0} & w^{0} \\
w^{0} & w^{1} & w^{2} & w^{3} & w^{4} \\
w^{0} & w^{2} & w^{4} & w^{1} & w^{3} \\
w^{0} & w^{3} & w^{2} & w^{4} & w^{2} \\
w^{0} & w^{4} & w^{3} & w^{2} & w^{2}
\end{array}\right)\left(\begin{array}{l}
a_{0} \\
a_{1} \\
a_{2} \\
a_{3} \\
a_{4}
\end{array}\right) .
\end{aligned}
$$


Algorithm:

$$
\begin{aligned}
& s_{1}=a_{1}+a_{4} \quad s_{2}=a_{1}-a_{4} \quad s_{3}=a_{3}+a_{2} \quad s_{4}=a_{3}-a_{2} \\
& s_{5}=s_{1}+s_{3} \quad s_{6}=s_{1}-s_{3} \quad s_{7}=s_{2}+I \cdot s_{4} \quad s_{8}=s_{2}-I \cdot s_{4} \\
& s_{9}=a_{0}+s_{5} \\
& m_{0}=1 \cdot s_{9} \\
& m_{1}=\left(\frac{\cos u+\cos 2 u}{2}-1\right) \cdot s_{5} u=\frac{2 \pi}{5} \\
& m_{2}=\frac{\cos u-\cos 2 u}{2} \cdot s_{6} \\
& m_{3}=\frac{i(\sin u+I \sin 2 u)}{2} \cdot s_{7} \\
& m_{5}=\frac{i(\sin u-I \sin 2 u)}{2} \cdot s_{8} \\
& s_{10}=m_{0}+m_{1} \quad s_{11}=s_{10}+m_{1} \\
& s_{12}=s_{10}-m_{2} \quad s_{13}=m_{4}+m_{3} \\
& s_{14}=m_{4}-m_{3} \quad s_{15}=s_{11}+s_{13} \\
& s_{16}=s_{11}-s_{13} \\
& s_{17}=s_{12}+I \cdot s_{14} \\
& s_{18}=s_{12}-I \cdot s_{14} \\
& A_{0}=m_{0} \quad A_{1}=s_{15} \quad A_{2}=s_{17} \quad A_{3}=s_{18} \quad A_{4}=s_{16}
\end{aligned}
$$

Mathematical Science Department IBM Thomas J. Watson Research Center Yorktown Heights, New York 10598

1. S. WINOGRAD, "Some bilinear forms whose multiplicative complexity depends on the field of constants," to be published in Mathematical Systems Theory, Vol. 10.

2. J. W. COOLEY \& J. W. TUKEY, "An algorithm for the machine calculation of complex Fourier series," Math. Comp., v. 19, 1965, pp. 297-301.

3. C. M. FIDUCCIA \& Y. ZALCSTEIN, Algebras Having Linear Multiplicative Complexities, Technical Report 46, Dept. of Computer Science, State University of New York, Stony Brook, August 1975.

4. A. L. TOOM, "The complexity of a scheme of functional elements simulating the multiplication of integers," Dokl. Akad. Nauk SSSR, v. 150, 1963, pp. 496-498 = Soviet Math. Dokl., v. 4, 1963, pp. 714-716.

5. C. M. RADER, "Discrete Fourier transforms when the number of data samples is prime," Proc. IEEE, v. 5, no. 6, June 1968, pp. 1107-1108.

6. I. J. GOOD, "The interaction of algorithm and practical Fourier series," J. Roy. Statist. Soc. Ser. B, v. 20, 1958, pp. 361-372; Addendum, v. 22, 1960, pp. 372-375. 